\title{
Paclitaxel Coated Balloon Fibroplasty: A more effective treatment of chronic symptomatic lead-related venous obstruction?
}

\author{
Omar Yacob ${ }^{1}$, Vijaywant Brar ${ }^{1}$, Arooje Towheed ${ }^{1}$, Cyrus Hadadi ${ }^{1}$, Susan O’Donoghue ${ }^{1}$, \\ and Seth Worley ${ }^{1}$ \\ ${ }^{1}$ MedStar Washington Hospital Center
}

November 26, 2020

\begin{abstract}
Subclavian venous obstruction is a common complication after cardiac device placement. Patients typically remain asymptomatic but at times may present with acute symptoms due to thrombus formation immediately after device placement or years later, due to chronic obstruction, with pain and swelling. Various techniques have been attempted to resolve symptoms in patients with chronic lead-related subclavian obstruction but are associated with a high recurrence of restenosis. Drug coated balloons (DCB) have been used subsequently after pre-dilation with high-pressure balloons, of the stenosed region, in patients with arteriovenous fistula obstruction, and have shown promising results. We discuss the utilization of DCB in our patient with chronic subclavian obstruction due to a permanent pacemaker lead.
\end{abstract}

Title: Paclitaxel Coated Balloon Fibroplasty: A more effective treatment of chronic symptomatic lead related venous obstruction?

Author: Omar Yacob MD ${ }^{1 a}$, Vijaywant Brar $\mathrm{MD}^{2 \mathrm{a}}$, Arooge Towheed MD ${ }^{2}$, Cyrus Hadadi $\mathrm{MD}^{2}$, Susan O'Donoghue $\mathrm{MD}^{2}$, Seth Worley MD ${ }^{2}$

\section{Affiliation:}

${ }^{1}$ MedStar Washington Hospital Center - Internal Medicine

${ }^{2}$ MedStar Washington Hospital Center - Division of Cardiac Electrophysiology

${ }^{a}$ Both authors contributed equally to the manuscript

Funding: None

Disclosure: None

Corresponding Author:

Omar Yacob, MD

MedStar Washington Hospital Center

Electrophysiology suite, Floor 5, 110 Irving St NW, Washington DC, 20010(USA)

omaryacob07@gmail.com

omar.yacob@medstar.net

Abbreviations: 
- Arteriovenous Fistula - AVF

- Biventricular Permanent Pacemaker - BiV-PPM

- Central Venous Catheter - CVC

- End Stage Renal Disease - ESRD

- Drug Coated Balloon- DCB

- Paclitaxel Coated Balloons- PCB

- Superior Vena Cava - SVC

Key teaching points:

- Lead related chronic fibrotic venous obstruction can occasionally be highly symptomatic

- Fibroplasty is a safe option that provides immediate relief however symptoms frequently recur presumably due to restenosis

- Drug coated balloons reduce restenosis in dialysis fistulas and may improve the long-term results in symptomatic patients with lead related chronic obstruction

\section{Introduction}

Upper extremity venous occlusion is a common complication encountered after transvenous lead placement that presents either acutely or chronically. Thrombus formation presents acutely with a painful, swollen upper extremity. Treatment can include catheter directed thrombolytics or anticoagulants/antiplatelet medications in an attempt to reduce thrombus burden and allow time for collateral formation (1)(2). Patients with acute symptoms that fail to resolve have undergone balloon dilation or subsequent stenting even though both techniques are associated with high rate of restenosis. Chronic lead related irritation of the venous endothelium results in neointimal hyperplasia with fibrosis. In many patients the lead related fibrosis eventually results in venous obstruction. Patients typically remain asymptomatic, due to formation of collateral circulation as fibrosis ensues (3) and the obstruction is typically discovered at the time of lead addition or replacement. Those who present with chronic symptoms presumably related to limited collaterals are sometimes managed with balloon dilation although symptoms quickly recur. The addition of stenting to balloon dilation has similar results in jailing and potential damage to the leads. The management of chronic lead related symptomatic venous obstruction is not well established. Herein we discuss the addition a paclitaxelcoated balloon (PCB) to high pressure balloon dilation as a potential way to improve the results in chronic symptomatic lead related venous obstruction

\section{Case repor $t$}

A 54-year-old woman with history of complete heart block status post biventricular permanent pacemaker (BiV-PPM) implantation, three years prior, presented with persistent left arm pain and swelling that resulted in limitations of her daily activities. Physical examination revealed edema above the clavicle, the arm and the hand. The remainder of the physical examination was unremarkable. Subclavian venogram revealed total occlusion of her left subclavian vein. Recognizing the limitations of balloon dilation alone and the risk and uncertain results of lead extraction followed by stenting an alternative strategy was sought. Given the favorable results of adding a drug coated balloon to high pressure balloon dilation in patients with stenotic dialysis fistulas we decided to add the drug coted balloon to high pressure balloon dilation.

\section{Procedural Technique:}

The procedure was performed, under general anesthesia in the electrophysiology lab. A left arm venogram was performed, which demonstrated total occlusion of the left subclavian vein with collateral formation (Figure: 1).

- The left axillary vein was cannulated using a 21-gauge micro puncture needle distal to the occlusion

- A 0.018 - inch micro-puncture wire was advanced to the left subclavian vein. The micro-puncture wire was unsuccessful in crossing the occlusion so further advanced techniques (4) were applied to cross the lesion 
- After crossing the lesion, subclavian fibroplasty was performed using a 4-mm non-compliant balloon from the superior vena cava-right atrium junction to the device pocket

- Multiple sites of severe stenosis of the left subclavian vein were noted

- After pre-dilatation, a PCB (7 X $40 \mathrm{~mm}$ ) was advanced to the site of initial total occlusion and the balloon was inflated for 30 seconds.

- The balloon was withdrawn \& hemostasis achieved

Post-operative Course:

The procedure was successfully performed in approximately 1 hour with no associated complications. Postoperatively the patient reported significant improvement in symptoms, with mild pain over the incision site. There was no damage to the leads \& the patient was discharged the following day after satisfactory pacing checks and chest X-ray.

\section{Discussion}

The current approach to chronic symptomatic lead related venous obstruction is unsatisfactory. Balloon dilation, to relieve the obstruction, results in symptom recurrence in a short duration and reintervention is required. Stenting without prior lead extraction is not considered to be an option. Even when leads are extracted, and stents deployed the results are not well defined. Given the apparent similarity between dilating a dialysis fistula and lead related venous obstruction the addition of a drug coated balloon to high pressure balloon dilation was considered a favorable option. Subclavian stenosis has been observed in approximately $15 \%$ of patients prior to device placement and increases up to $50 \%$ after transvenous manipulation (5). Although many factors are involved in the development of vascular occlusion the two primary processes involved are: thrombus formation and neointimal hyperplasia with subsequent fibrosis. In the acute settings thrombus formation around the lead can lead to acute symptoms for which anticoagulative agents are often used to reduce the risk of embolic complications while collaterals develop. Lead placement also irritates the venous endothelium inducing an inflammatory cascade leading to excessive proliferation of the connective tissue resulting in a fibrotic occlusion (5).

Multiple strategies have been attempted for patients with chronic symptomatic lead related venous obstruction. Surgical approaches predominated in the 1990s until the advent of percutaneous techniques and subsequent introduction of balloon dilation. Riley et al (6) conducted the first retrospective pooled analysis of 104 patients with symptomatic lead associated superior vena cava syndrome and reported that the most common treatment approach superior with the lowest rate of restenosis. However stenting results in compression of the lead between the stent and the wall of the vein referred to as 'jailing' of the lead. 'Jailing' can compromise lead integrity and/or result in fracture. Jailing also complicates lead removal in the cases of infection. The current Heart Rhythm Society expert consensus is to extract transvenous leads before stenting, to avoid their entrapment (7).

In the Mayo clinic (8) six patients identified with lead associated symptomatic superior vena syndrome had stent implantation performed after successful lead removal followed by reimplantation of the leads however some patients required reintervention. Anticoagulation and antiplatelet therapy have also been utilized in chronic occlusions but have produced conflicting results, with majority studies reporting that anticoagulant or antiplatelet use was not associated with a decreased incidence of venous stenosis or thrombosis (2)(9) due to their ineffectiveness on chronic fibrotic lesions, that often exist in these patients, rather than atherosclerotic lesions (6). In a study done at Cleveland Clinic (10) 861 device leads were histologically examined after extraction, in patients with leads for $>1$ year, and examination of tissue demonstrated dense fibrous tissue with the presence of calcification. From these histological findings the nature of the tissue involved can help guide further treatment.

Worley et al (11) performed balloon fibroplasty in 371 asymptomatic patients with significant subclavian stenosis to facilitate lead revision or upgrade demonstrating the safety and practicality of subclavian balloon fibroplasty, using non-complaint balloons. Balloon dilation is the standard treatment in end stage renal disease (ESRD) patients with stenosis of their arteriovenous fistulas (AVF). Compared to standard non- 
compliant balloons (Rate Burst Pressure 20 ATM) ultra-noncompliant high-pressure balloons (Rate Burst Pressure 30 ATM) improve fistula patency. However even with high pressure balloons (12)(13) durability was limited thus stenting was added in an attempt to prolong patency. However even stents for AVF were associated with a high risk of restenosis. Due to the meager long terms successes of balloon angioplasty and vascular stents Kitrou et al (14) and Lookstein et al (15) studied the effect of PCBs on dysfunctional AVF. Because of their low rated burst pressure, PCBs, required pre-dilation with high pressure balloons to dilate the hard stenotic and rigid lesions in the vasculature. Adding PCB demonstrated superiority to both non-compliant balloons and high-pressure balloons in maintaining target vessel patency at 6 months with non-inferior primary safety end points. PCBs also significantly extended the event-free period for the AVF circuit. Presumably, deliverance of local drug-delivery device inhibits the process of neointimal hyperplasia and enabled a longer intervention-free period.

The stenosis observed in an AVF is due to endothelial irritation leading to tissue proliferation and subsequent fibrosis. It is likely that the fibrotic occlusion associated with chronic leads is similar to that observed in patients with AVF. Our report describes the first addition of DCB for subclavian fibroplasty in a symptomatic patient with chronic transvenous leads. Because DCBs have been proven safe and effective in maintaining patency of stenotic dialysis fistulas (16)(17) they may help improve long term patency in symptomatic lead related obstruction. When fibroplasty is performed for access in asymptomatic patients with chronic leads the vein quickly reocclude. The addition of a PCB balloon may be useful in maintaining patency.

To know for certain whether PCB improve patency in chronic symptomatic patients with lead related obstruction requires a randomized trial. However, given the limited alternatives it is important to consider the addition of a PCB to balloon dilation as an option for patients with symptomatic venous obstruction before resorting to extraction and stenting. PCB may also prove useful in maintaining patency in asymptomatic patients requiring fibroplasty for lead replacement or upgrade.

\section{Acknowledgments}

Dr. Worley receives has in the past received compensation in various forms from Medtronic. He currently receives compensation in various forms including royalties from Merit Medical, Pressure Products, Guidant, and St. Jude Medical. No financial support was provided for the creation of this case report.

\section{Reference:}

1. Rooden CJV, Molhoek SG, Rosendaal FR, Schalij MJ, Meinders AE, Huisman MV. Incidence and Risk Factors of Early Venous Thrombosis Associated with Permanent Pacemaker Leads. J Cardiovasc Electrophysiol. 2004;15(11):1258-62.

2. Abu-El-Haija Basil, Bhave Prashant D., Campbell Dwayne N., Mazur Alexander, Hodgson-Zingman Denice M., Cotarlan Vlad, et al. Venous Stenosis After Transvenous Lead Placement: A Study of Outcomes and Risk Factors in 212 Consecutive Patients. J Am Heart Assoc. 4(8):e001878.

3. Stoney WS, Addlestone RB, Alford WC, Burrus GR, Frist RA, Thomas CS. The Incidence of Venous Thrombosis Following Long-Term Transvenous Pacing. :5.

4. Zou F, Brar V, Worley SJ. Interventional device implantation, Part I: Basic techniques to avoid complications: A hands-on approach. J Cardiovasc Electrophysiol. 2020 Sep 18;

5. Spittell PC, Hayes DL. Venous Complications After Insertion of a Transvenous Pacemaker. Mayo Clin Proc. 1992 Mar 1;67(3):258-65.

6. Riley RF, Petersen SE, Ferguson JD, Bashir Y. Managing superior vena cava syndrome as a complication of pacemaker implantation: a pooled analysis of clinical practice. Pacing Clin Electrophysiol PACE. 2010 Apr;33(4):420-5.

7. Bongiorni MG, Burri H, Deharo JC, Starck C, Kennergren C, Saghy L, et al. 2018 EHRA expert consensus statement on lead extraction: recommendations on definitions, endpoints, research trial design, and data 
collection requirements for clinical scientific studies and registries: endorsed by APHRS/HRS/LAHRS. EP Eur. 2018 Jul 1;20(7):1217-1217.

8. Fu H-X, Huang X-M, Zhong L, Osborn MJ, Bjarnason H, Mulpuru S, et al. Outcome and Management of Pacemaker-Induced Superior Vena Cava Syndrome: PACEMAKER-INDUCED SUPERIOR VENA CAVA SYNDROME. Pacing Clin Electrophysiol. 2014 Nov;37(11):1470-6.

9. Goto Y, Abe T, Sekine S, Sakurada T. Long-Term Thrombosis after Transvenous Permanent Pacemaker Implantation. Pacing Clin Electrophysiol. 1998;21(6):1192-5.

10. Tarakji KG, Saliba W, Markabawi D, Rodriguez ER, Krauthammer Y, Brunner MP, et al. Unrecognized venous injuries after cardiac implantable electronic device transvenous lead extraction. Heart Rhythm. 2018 Mar;15(3):318-25.

11. Worley SJ, Gohn DC, Pulliam RW, Raifsnider MA, Ebersole BI, Tuzi J. Subclavian venoplasty by the implanting physicians in 373 patients over 11 years. Heart Rhythm. 2011 Apr 1;8(4):526-33.

12. Beathard GA. The Treatment of Vascular Access Graft Dysfunction: A Nephrologist's View And Experience. Adv Ren Replace Ther. 1994 Jul 1;1(2):131-47.

13. Buriánková E, Köcher M, Bachleda P, Utíkal P, Kojecký Z, Černá M, et al. Endovascular treatment of central venous stenoses in patients with dialysis shunts. Biomed Pap. 2003 Dec 1;147(2):203-6.

14. Kitrou PM, Papadimatos P, Spiliopoulos S, Katsanos K, Christeas N, Brountzos E, et al. PaclitaxelCoated Balloons for the Treatment of Symptomatic Central Venous Stenosis in Dialysis Access: Results from a Randomized Controlled Trial. J Vasc Interv Radiol. 2017 Jun;28(6):811-7.

15. Lookstein RA, Haruguchi H, Ouriel K, Weinberg I, Lei L, Cihlar S, et al. Drug-Coated Balloons for Dysfunctional Dialysis Arteriovenous Fistulas. N Engl J Med 2020 Aug 19 doi/10.1056/NEJMoa1914617

16. Massmann A, Fries P, Obst-Gleditsch K, Minko P, Shayesteh-Kheslat R, Buecker A. Paclitaxel-Coated Balloon Angioplasty for Symptomatic Central Vein Restenosis in Patients With Hemodialysis Fistulas. J Endovasc Ther. 2015 Feb 1;22(1):74-9.

17. Kennedy SA, Mafeld S, Baerlocher MO, Jaberi A, Rajan DK. Drug-Coated Balloon Angioplasty in Hemodialysis Circuits: A Systematic Review and Meta-Analysis. J Vasc Interv Radiol. 2019 Apr 1;30(4):483494.e1. 

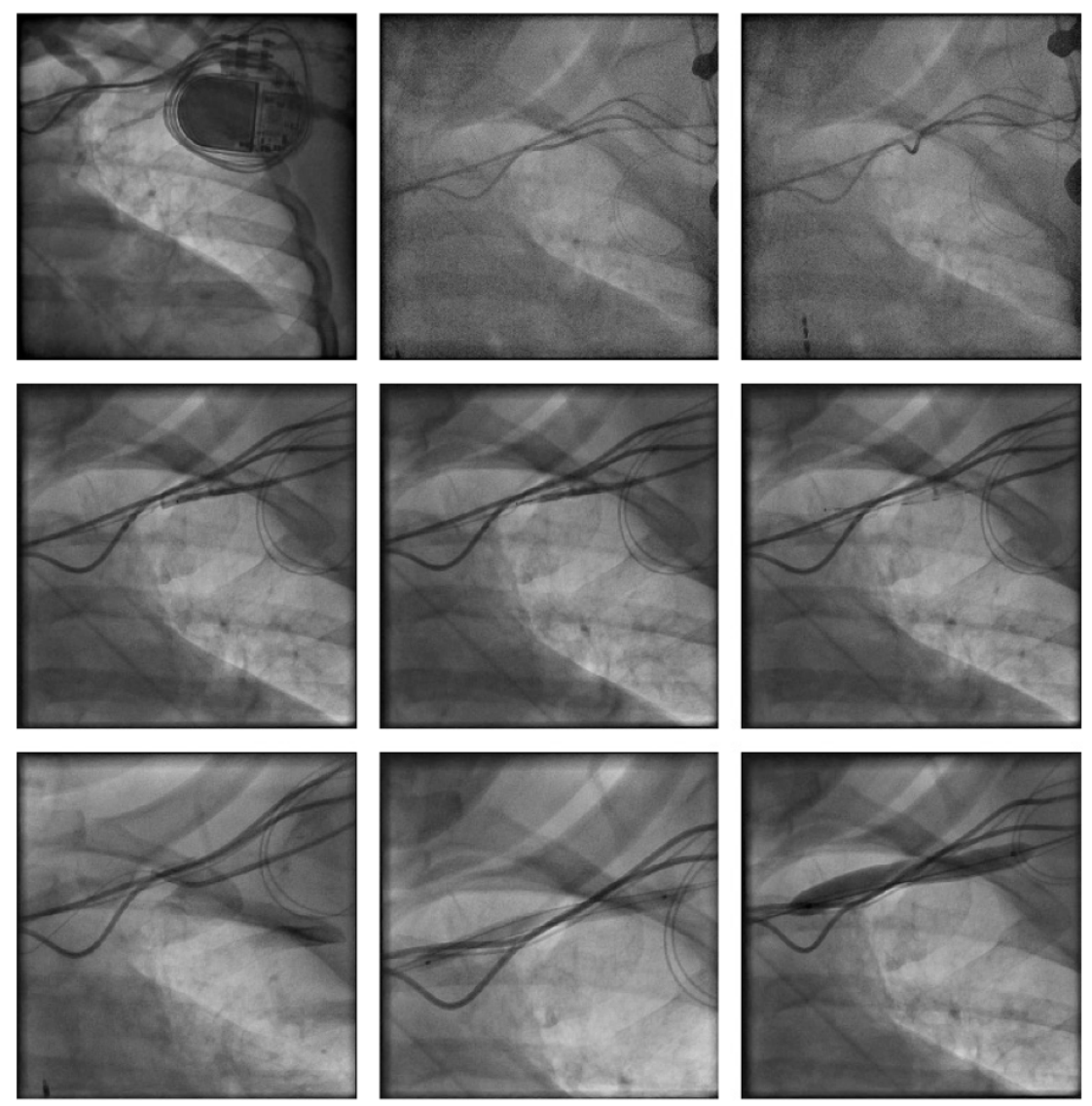